\title{
Article
}

\section{Emotional resilience and event centrality mediate posttraumatic growth following adverse childhood experiences}

Tranter, Heidi, Brooks, Matthew and Khan, Roxanne

Available at https://clok.uclan.ac.uk/33661/

Tranter, Heidi, Brooks, Matthew and Khan, Roxanne orcid iconORCID: 00000002-3485-2450 (2021) Emotional resilience and event centrality mediate posttraumatic growth following adverse childhood experiences. Psychological Trauma: Theory, Research, Practice, and Policy, 13 (2). pp. 165-173. ISSN 1942-9681

It is advisable to refer to the publisher's version if you intend to cite from the work. http://dx.doi.org/10.1037/tra0000953

For more information about UCLan's research in this area go to http://www.uclan.ac.uk/researchgroups/ and search for <name of research Group>.

For information about Research generally at UCLan please go to http://www.uclan.ac.uk/research/

All outputs in CLoK are protected by Intellectual Property Rights law, including Copyright law. Copyright, IPR and Moral Rights for the works on this site are retained by the individual authors and/or other copyright owners. Terms and conditions for use of this material are defined in the policies page.

\section{CLoK}

Central Lancashire online Knowledge www.clok.uclan.ac.uk

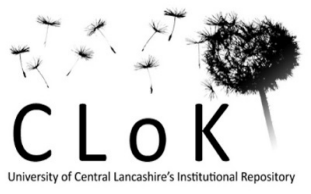


Recommended citation: Tranter. H., Brooks, M., \& Khan, R. (2020, in press). Emotional resilience and turning points mediate growth following adverse childhood experiences. Psychological Trauma: Theory, Research, Practice, and Policy.

Emotional resilience and event centrality mediate posttraumatic growth following adverse childhood experiences

\section{Heidi Tranter, MSc}

University of Central Lancashire, School of Psychology, UK

\section{Matthew Brooks, PhD*}

Manchester Metropolitan University, Department of Psychology, UK

ORCID: 0000-0002-5469-7769

\section{Roxanne Khan, PhD}

University of Central Lancashire, School of Psychology, UK

ORCID: 0000-0002-3485-2450

*Corresponding Author:

Dr. Matthew Brooks

Department of Psychology

Brooks Building

Manchester Metropolitan University

Manchester

M15 6GX

United Kingdom

Email: $\underline{\text { m.brooks@mmu.ac.uk }}$

Other author contact details:

Heidi Tranter- $\underline{\text { HRTranter@uclan.ac.uk }}$

Dr. Roxanne Khan-rkhan2@uclan.ac.uk 


\begin{abstract}
Research has long established that adverse childhood experiences (ACEs) can lead to a range of negative psychological consequences, including post-traumatic stress (PTS) symptoms. It is also increasingly recognized that ACEs can serve as a catalyst for positive changes, such as post-traumatic growth (PTG). The mechanisms by which people report negative or positive changes are less well known. This study explored whether emotional resilience and event centrality could determine the degree of negative or positive changes reported following ACEs. Participants ( $N=167 ; 54.5 \%$ female; aged 19-95 years) completed an online survey measuring experiences of childhood adversity, resilience, event centrality, PTS symptoms and PTG. Mediation analyses indicated that resilience and event centrality explained PTG only, exerting significant medium negative and small positive indirect effects on PTG, respectively. These findings indicate that following ACEs, the treatment and management of emotional resilience and event centrality could lead to positive effects on psychological well-being.
\end{abstract}

Keywords: adverse childhood experiences; event centrality; post-traumatic growth; post-traumatic stress; resilience

Clinical impact statement: The study findings indicate that following adverse childhood experiences, high levels of resilience and viewing the experience as a positive identity change may determine the degree of positive changes reported. Support and interventions could focus on enhancing resilience and awareness of positive identity changes to encourage survivors to recognise gains from their childhood experiences. 


\section{Introduction}

A large body of research has improved our understanding of the psychological impact of being exposed to adverse childhood experiences (ACEs; i.e. emotional, physical and sexual abuse or neglect, and family dysfunction; Bryan, 2019; Merrick et al., 2017). In the general non-clinical population, the prevalence of ACEs can vary, with 30.9\% of students (Bufford et al., 2017) and 66.9\% of community-based adults (Thomson \& Jacque, 2017) endorsing at least one or more ACEs. The negative physical, psychological and social changes associated with ACEs have been documented to persist from childhood through to adulthood (Kalmakis \& Chandler, 2015), and can include depression (Chapman et al., 2004), post-traumatic stress (PTS) symptoms (Kalmakis et al., 2019), chronic health conditions (Monnat \& Chandler, 2015; Sheffler et al., 2020), and an increased propensity to experience negative outcomes in adulthood (Bryan, 2019; Felitti et al., 1998). While these negative outcomes are an important focus of research efforts, studies have increasingly shown that ACEs can serve as a catalyst for positive changes (e.g., Brooks et al., 2019; Sheridan, \& Carr, 2020; Tedeschi \& Calhoun, 2004), which also merits further investigation.

PTS symptoms are commonly explored as a potential negative psychological consequence of exposure to ACEs. PTS symptoms are characterised by avoidance, hyperarousal, intrusive thoughts, and negative changes in mood (Kilpatrick et al., 2013). These symptoms can present in the aftermath of a variety of ACEs, such as physical abuse or neglect (Barlow et al., 2017), which has the potential to negatively influence an individual's life trajectory. For instance, PTS symptoms from prior ACES could lead to an increased risk of victimization and more frequent encounters with subsequent adverse events (Westfall \& Nemeroff, 2018). Research (e.g., Draucker et al, 2011) has also speculated that adverse events can simultaneously lead to enhanced psychological functioning following ACEs, alongside negative changes. Indeed, the ability for people to endorse positive psychological changes in the aftermath of ACEs has received increasing empirical attention, one such 
RESILIENCE, EVENT CENTRALITY AND GROWTH

change being post-traumatic growth (PTG; e.g., Nelson et al., 2019; Schaefer et al, 2018; Woodward \& Joseph, 2003; Zeidner \& Kampler, 2020).

PTG refers to positive changes that emerge from the psychological struggle with stressful life events (Tedeschi \& Calhoun, 2004). Typical changes include a renewed appreciation for life, a new life philosophy, and improved social relationships. Critical to the development of PTG is the role of intrusive processing, which is a core feature of PTS symptoms (Kilpatrick et al., 2013). The distress that ensues from making sense of the adverse event is thought to drive the cognitive processing needed to experience positive transformation (Tedeschi \& Calhoun, 2004). Recent studies have reported PTG in survivors of child sexual abuse (Sheridan \& Carr, 2020) and neglect (Brooks et al., 2019), suggesting that people do have the ability to perceive some benefits arising from their ACEs, which warrants further investigation.

While negative and positive outcomes have been documented in response to ACEs, the mere experience of adversity alone does not lead to PTS or PTG. Indeed, research has advanced possible mediating variables that need to present for such outcomes to be demonstrated. One variable of interest is the notion of 'event centrality', which refers to an event(s) in an individual's life that alter their life trajectory in a significant way (Tavernier \& Willoughby, 2012). These events may represent important life changes that can act as a reference point for expectations or attributions and can alter people's self-perceptions (Berntsen \& Rubin, 2007). It has been argued that individuals have a need to maintain coherence and consistency in their lives (Clausen, 1995). ACEs are a potential challenge to this need, and so event centrality may lead to negative consequences, such as the development of PTS symptoms (Robinaugh \& McNally, 2011), and low self-ratings of physical health (Sutin et al., 2010). However, positive changes from central events have also been observed, such as the ability to find meaning in events, a characteristic of PTG (Groleau et al., 2013). It may be that event centrality could also serve as an opportunity for learning and potential positive change, as well as negative outcomes. 
Alongside event centrality, research has also considered the role of emotional resilience and its influence on psychological adjustment following ACEs. Resilience is defined as a dynamic characteristic which allows some individuals to experience a relatively positive outcome, despite stressful or adverse experiences (Rutter, 2013; Stainton et al., 2019). When considering childhood maltreatment, individuals who have greater individual resilience are less likely to experience depression, PTS and other negative consequences associated with adversity (Kaloeti et al., 2019; Meng et al., 2018). It may be that resilience serves to 'buffer' individuals against negative consequences associated with ACEs (Foster, 2018), and so it follows that individual resilience could determine the degree of PTS experienced.

While resilience could exert buffering effects on negative symptoms, it may simultaneously enhance the likelihood of PTG. The salutogenic nature of emotional resilience and PTG emphasises strength in individuals to overcome adversity and follow new life trajectories (Infurna \& Jayawickreme, 2019). Despite sharing similar characteristics, literature has determined that resilience and PTG are distinct concepts, with resilience referring to a cluster of characteristics that help people overcome adversity, and growth indicating improvements to psychological functioning (Levine et al., 2009). It may be that resilient individuals are less likely to experience PTG, as they are more easily able to mitigate the negative effects of their ACEs and not experience the emotional struggle needed for growth to occur. There are limited investigations of the mediating role of emotional resilience on PTG following ACEs, although research in response to school shootings suggests it could act as a protective factor (Vieselmeyer et al., 2017). Furthermore, this study will extend on the findings of previous investigations (e.g., Goodman et al., 2017) that have focused on isolated events by exploring the influence of multiple exposures to ACEs.

\section{Aims of Study}

Overall, ACEs can present significant psychological challenges to individuals. Depending on event centrality, these life events confront an individual's sense of identity that is subsequently 
conducive to positive (PTG) or negative (PTS) changes. Equally, ACEs may impact on individual resilience in positive or negative ways, which in turn can affect perceptions of positive or negative change. The aim of this study, therefore, is to determine whether emotional resilience and event centrality influence the likelihood of PTG and PTS following ACEs. It is hypothesized that ACEs could lead to changes in resilience and represent central events which will impact on the degree of PTG or PTS symptoms reported.

\section{Method}

\section{Participants}

A general population sample of 167 participants (54.5\% female) were recruited using opportunity sampling. The age of participants ranged from 19 to 95 years $(M=38.19, S D=15.90)$. Table 1 shows the trauma history of participants, with prevalence of subtypes of childhood maltreatment and household dysfunction. ACE scores in this study ranged from $0-12(M=2.37, S D$ $=2.87)$, suggesting that on average, participants in this study had low exposure to adverse events.

\section{[INSERT TABLE 1 ABOUT HERE]}

\section{Measures}

Adverse Childhood Experiences. An amended version of the Adverse Childhood Experiences Questionnaire (ACE-Q; Felitti et al., 1998) was used to measure an individual's exposure to abuse and/or neglect during childhood. In this study, some items from the original questionnaire were collapsed and each statement was able to be scored on an individual basis, rather than participants indicating whether they had experienced one of multiple items under each subcategory in the original measure. Example items include, 'Did a parent or other adult in the household often or very often swear at you, insult you, put you down or humiliate you?' Participants were asked to respond to each item and were scored as 0 (no) or 1 (yes) and the overall ACE score was calculated, the 
highest possible score being 16 . The ACE-Q was originally designed to be used as a checklist, but in this study it was used as a scale. The amended ACE-Q was found to have good internal consistency $(\alpha=.82)$, which exceeded previous studies that have used it as a scale measure (e.g. $\alpha=.77$, Bufford et al., 2017).

Emotional Resilience. The Connor-Davidson Resilience Scale (Campbell-Sills \& Stein, 2007) is a 10-item scale measuring individual levels of emotional resilience. Items include, 'I tend to bounce back after illness, injury or other hardships.' Participants were asked to determine the extent to which they related to each statement by selecting a response using a scale from 0 (never true) to 4 (very true). Internal consistency for the measure was high $(\alpha=.93)$, exceeding the original value reported by Campbell-Sills and Stein $(2007 ; \alpha=.85)$.

Event Centrality. The Centrality of Events Scale - Short Form (CES; Bernsten \& Rubin, 2006) is a 7-item measure of an individual's perception of an event in which they felt a decisive change was made. Items include, 'This event has become a reference point for the way I understand myself and the world.' Participants determined the extent to which they agreed with each statement by selecting the most appropriate response on a scale of 0 (totally disagree) to 4 (totally agree). The CES demonstrates high internal consistency ( $\alpha=.88$; Bernsten \& Rubin, 2006), which was reflected in the current study $(\alpha=.90)$.

Post-traumatic Stress. The Primary Care PTSD Screen for DSM-5 (PC-PTSD-5; Prins et al., 2016) is a 5-item screening measure of PTSD symptoms. Example items include, 'In the past month, have you had nightmares about the event(s) or thought about the event(s) when you did not want to?' and are scored 0 (no) or 1 (yes). The PC-PTSD- 5 was originally designed to be a checklist but was used as a scale in this study, with higher scores are indicative of greater PTSD symptoms. The measure demonstrates high diagnostic accuracy and face validity (Prins et al., 2016). In this study, the measure was found to have good internal consistency $(\alpha=.83)$. 
Post-traumatic Growth. The Post-Traumatic Growth Inventory - Short Form (Cann et al., 2010) is a 10-item measure of potential positive changes arising from adverse events. Participants determined the extent to which they agreed with each statement by selecting the relevant response on a scale from 0 (totally disagree) to 4 (totally agree), with items such as, 'I am able to do better things with my life.' The PTGI-SF has high internal consistency $(\alpha=.90)$, with similar reliability to the full 21-item version (Cann et al., 2010), and the internal consistency found in the current study ( $\alpha=$ $.91)$.

Procedure

Participants were invited to complete an online survey of their ACEs and potential positive and negative consequences, which was accessed through a weblink that provided information about the research. Upon providing informed consent, participants were asked to complete the survey. If the participant withdrew from the study at any point, they were redirected to the debrief which contained information on support services. The study received institutional ethical approval.

\section{Data Analysis}

The data analysis consisted of two phases. First, Pearson's correlations examined the direction and magnitude of associations among key study variables. Second, mediation analyses were conducted to determine indirect effects with 5000 bootstrapped samples and bias-corrected 95\% confidence intervals (BCa Cl) using the SPSS PROCESS macro (Hayes, 2013). Analysis of indirect effects is advantageous compared to casual steps analysis as it does not require all paths to be significant (Hayes, 2013). Confidence intervals that do not contain zero indicate a significant indirect effect, and thus mediation. Standardised regression coefficients ( $\beta$ ), direct and completely standardised indirect effects $\left(a b_{c s}\right)$ are reported in line with current recommendations (Hayes, 2013). Statistics for the total effect model are also reported, which refer to the influence of the predictor $(X)$ on the outcome $(Y)$ with no mediators present in the model (Hayes, 2013). Effect sizes are 
RESILIENCE, EVENT CENTRALITY AND GROWTH

identified as small ( $a b_{c s}$ of .01 to .08 ), medium ( $a b_{c s}$ of .09 to .24 ) or large ( $a b_{c s} \geq .25$; Preacher \& Kelly, 2011). To conform with recommendations for cross-sectional models of indirect effects (Preacher \& Hayes, 2004), reverse mediation analyses were also conducted to confirm the specification and directionality of the relationships. In these models, the outcome variables (PTS and PTG) were substituted for the mediators (Event Centrality and Emotional Resilience).

\section{Results}

\section{Descriptive and Correlational Statistics}

Means and standard deviations for the key study variables are reported in Table 2. Data indicated that the average ACE score was relatively low for the sample, with participants recording approximately two separate ACEs. Skewness and kurtosis values indicated all variables fell within acceptable parameters (up to an absolute value of 1.96) for normality (Tabachnick \& Fidell, 2013). In the sample, $38.9 \%$ recorded no ACEs, $13.2 \%$ reported one ACE, $14.4 \%$ reported two ACEs, $6 \%$ recorded three ACEs, and $23.4 \%$ reported $\geq 4$ ACEs.

\section{[INSERT TABLE 2 ABOUT HERE]}

A correlational analysis was conducted to determine significant relationships between variables (see Table 3). Higher ACE scores were negatively and significantly related to emotional resilience and PTS symptoms. Emotional Resilience was positively associated with Event Centrality, PTS symptoms and PTG. Event Centrality was negatively and significantly correlated with PTS symptoms, and positively associated with PTG.

[INSERT TABLE 3 ABOUT HERE]

\section{Mediation Analyses}


Two mediation analyses were conducted using multiple mediators, with the findings presented in Table 4. In the first mediation model, the total number of ACEs was entered as the independent variable, Emotional Resilience and Event Centrality were entered simultaneously as mediators, and PTS symptoms as the dependent variable. This model accounted for $56.2 \%$ of the variance in PTS symptoms $[F(3,163)=69.62, p<.001]$. The direct effect of ACEs on PTS symptoms was significant $(p<.001)$. Both of the mediators' upper confidence intervals included zero, and thus neither exerted significant indirect effects on the relationship between ACEs and PTS symptoms. The total effect model was significant, $\left[\beta=-.73,95 \% \mathrm{BCa} \mathrm{Cl}[-.48,-.36], R^{2}=.53\right]$ and indicated that ACEs significantly and negatively predicted PTS symptoms $(p<.001)$.

The second mediation analysis consisted of ACE scores as the independent variable, Emotional Resilience and Event Centrality entered simultaneously as mediators, and PTG as the dependent variable. This multiple mediation model explained $38.4 \%$ of the variance in PTG $[F(3$, $163)=33.90, p<.001]$. The direct effect of ACEs on PTG was not significant $(p=.636)$. However, the indirect path through Emotional Resilience demonstrated a medium negative effect that was significant. Event Centrality also significantly mediated the relationship between ACEs and PTG, exerting a small positive effect. The total effect model was not significant, $[\beta=.00,95 \% \mathrm{BCa} \mathrm{Cl}[-.49$, $\left..46], R^{2}=.00\right]$, with ACEs not significantly predicting PTG when mediators were absent $(p=.957)$.

\section{[INSERT TABLE 4 ABOUT HERE]}

The results of the reverse mediation analyses indicated that PTS symptoms was the sole mediator of Emotional Resilience and Event Centrality. The mediation model with ACE score as the independent variable, Event Centrality, PTS symptoms and PTG as mediators, and Emotional Resilience as the dependent variable, accounted for $28.9 \%$ of the variance in Emotional Resilience [ $F$ $(4,162)=16.47, p<.001]$. The direct effect was not significant $(p=.282)$, and the only significant indirect effect was found for PTS symptoms [a $\left.b_{\mathrm{cs}}=-.16, \mathrm{BCa} \mathrm{Cl}:-.29,-.04\right]$, demonstrating a negative medium effect. Finally, the mediation model with ACE score as the independent variable, Emotional 
Resilience, PTS symptoms and PTG as mediators, and Event Centrality as the dependent variable, accounted for $31.6 \%$ of the variance in Event Centrality $[F(4,162)=16.47, p<.001]$. The direct effect was not significant $(p=.920)$, and again, the only significant indirect effect was observed for PTS symptoms $\left[a b_{c s}=.17, \mathrm{BCa} \mathrm{Cl}: .04, .30\right]$, which indicated a positive moderate effect.

\section{Discussion}

Research studies have begun to recognize that exposure to adversity alone is not sufficient to explain differential psychological outcomes (e.g., Bernard et al., 2015; Brooks et al., 2019). This study aimed to determine whether emotional resilience and event centrality influence the likelihood of PTG and PTS following ACEs. It was hypothesized that ACEs could lead to changes in resilience and represent central events that impact on the degree of PTG or PTS symptoms reported. Findings indicated that resilience and event centrality were significant mediators of positive (PTG) but not negative (PTS) outcomes.

The sample reported a low frequency of ACEs $(M=2.37)$ on the amended ACE questionnaire, although it is common for individuals to report more than one ACE (Felitti et al., 1998; Seery et al., 2010). Although the measure was amended which limits direct comparisons, ACE frequency in this study exceeded averages in samples of students ( $M=1.41$; Bufford et al., 2017), was similar to adults recruited from primary care clinics ( $M=2.11$; Framptom et al., 2018), but was below that of that adult females fostered as children $(M=5.60 ;$ Bruskas \& Tessin, 2013), all of which are studies that used the original ACE measure. While the prevalence of ACEs was low, the sample recorded a high frequency of PTS symptoms $(M=3.74)$ which was greater than those observed in chronic pain patients ( $M=1.40$; Langford et al., 2018) and female former military veterans ( $M=$ 1.47; Nillni et al., 2020) using the same PC-PTSD-5 measure.

\section{Emotional Resilience and Event Centrality as Mediators of Posttraumatic Growth Following}

\section{Adverse Childhood Experiences}


Consistent with other research (Duan et al., 2015), results showed that resilience was positively correlated with PTG, indicating that individuals with higher emotional resilience may also report more PTG. This may not be surprising, given that the salutogenic nature of emotional resilience and PTG both emphasise strength in individuals to overcome adversity (Infurna \& Jayawickreme, 2019). Research has found that resilient individuals are more likely to overcome adversity, and as a result, demonstrate enhanced psychological functioning (Levine et al., 2009). Yet, the negative indirect effect in the mediation analysis suggests that people low in resilience are more likely to experience PTG, which points to a more nuanced relationship than suggested by the correlational analysis alone. This finding aligns with theorized relationships in that some degree of emotional struggle is needed to experience PTG (Tedeschi \& Calhoun, 2004); if survivors are not challenged by their ACEs due to the buffering effect of resilience, then there is less impetus to grow. Given that PTG did not mediate resilient outcomes in the reverse mediation analysis, it may be that resilience and growth are related, yet distinct, salutogenic constructs (Weber et al., 2019), although future prospective studies could provide more insight into this relationship.

Alongside resilience, this study found that event centrality positively mediated the relationship between ACEs and PTG. Studies (e.g., Brooks et al., 2017; Groleau et al., 2013) have indicated that central events can lead to both positive and negative outcomes, by motivating cognitive processes that lead survivors to strongly identify with their experiences. For some survivors, event centrality may signal more deliberate attempts to contemplate meaning behind events (Easton, 2013), while others may attach a negative valance to their ACEs, viewing themselves as a 'victim' primarily or solely, and thus becoming consumed by their experiences (Bernard et al., 2015; Groleau et al., 2013).

\section{Emotional Resilience, Event Centrality and Posttraumatic Stress Following Adverse Childhood}

\section{Experiences}


Unlike the PTG mediation model, resilience and event centrality did not explain the relationship between ACEs and PTS symptoms. This result is inconsistent with some prior research in this area that finds event centrality to be positively associated with PTS symptoms (Brooks et al., 2017; Groleau et al., 2013; Robinaugh \& McNally, 2011), and resilience to be negatively related to PTS (Thompson et al., 2018). It may be that for some participants, their ACEs were not perceived as significant central events in their lives. Consequently, some people may not be challenged by their ACEs, and thus it would seem unlikely it would negatively impact on levels of resilience or mark a significant central event that would propel the individual to experience distress.

In addition to event centrality, emotional resilience did not explain the relationship between ACEs and PTS symptoms. This finding is contrary to the idea that resilience serves to 'buffer' individuals against negative consequences associated with ACEs (Foster, 2018). However, other variables may exert greater effects on PTS symptoms than individual levels of resilience. Studies have indicated that perceived social support (Aydin et al., 2016), poor mental health (Machisa et al., 2016) and depression (Aydin et al., 2016), contribute to the onset of PTS symptoms following ACE exposure. Therefore, future research should explore how these factors could mediate the development of PTS and PTG in the aftermath of ACEs.

The reverse mediation analyses revealed that PTS symptoms explained relationships between ACEs and resilience, as well as ACEs and event centrality. These findings are consistent with the literature that attests to the potential dual function of PTS symptoms as both a marker of distress and a characteristic of adaptive cognitive processing efforts that can lead to both negative and positive changes in identity perception (Brooks et al., 2019; Groleau et al., 2013). Recent research has also found that intrusive symptoms, a characteristic of PTS, can mediate associations between ACEs and resilience (Rawlins et al., in press). The results offer further support to the idea that resilience may also be an outcome of the psychological struggle with ACEs (Olsson et al., 2015). 
It may be that integrating memories of adverse events do not always contribute towards the experience of trauma, as previously thought (e.g., Halligan et al., 2003).

The study did find some seemingly counterintuitive results from the correlations observed between PTS symptoms and other variables in the study. The negative correlation between ACEs and PTS symptoms, appears to go against common understandings that more frequent ACEs are associated with increased symptom complexity (e.g., Schalinski et al., 2016). This may be due to individuals in the sample minimising symptoms, or the fact that the sample had relatively few ACE exposures thus contributing to the low reported PTS symptoms. This study extended previous literature that focuses on single types of ACEs (e.g., Goodman et al., 2017) to account for multiple exposures that people often encounter. Individuals exposed to repeated traumatic events may exhibit traits associated with emotional numbing (Kerig et al., 2012). It is possible this may be an attempt to reduce the distress associated with multiple ACEs, and so the individual may not report exacerbated symptoms in response to repeated exposure. PTS symptoms were also positively related to resilience, and negatively related to event centrality, in directions contrary to prior findings in this area (e.g., Brooks et al., 2017; Duan et al., 2015). One explanation may be the presence of non-linear relationships between these variables, such that while some degree of adversity is beneficial, too much would be overwhelming for individuals. For instance, research has suggested PTG and PTS symptoms demonstrate stronger curvilinear than linear relationships which vary according to age and trauma type (Shakespeare-Finch \& Lurie-Beck, 2014). While most people in the currenty experienced relatively few ACEs, almost a quarter (23.4\%) reported four or more events. It may be that relationships between resilience, event centrality, PTS and PTG vary according to the degree of adversity experienced, which future research could investigate.

\section{Limitations}

While this study contributed to understanding the psychological mechanisms responsible for PTS and PTG development following ACEs, it is not without limitation. The use of a clinical sample 
would enable researchers to better explore how event centrality and resilience influence PTS and PTG in a population who experience a high proportion of ACEs to see if similar relationships are observed. Additional demographic data were not collected in respect of ethnicity, location or current mental health diagnoses, which presents some limitations in terms of the generalisability of the findings. Furthermore, the study used a retrospective design when assessing ACE reports, which may be subject to memory fallibility over time. Future studies should therefore use prospective methods to establish causality among the relationships identified in this study. Finally, while the PTGI measure employed in this study is widely used (Cann et al., 2010), it is difficult to determine how much of that positive change is solely attributable to a specific adverse event, or whether the change reflects genuine improvements in wellbeing, or merely whether the participant believes they have changed (Jayawickreme \& Blackie, 2014).

\section{Implications}

Findings from this study indicated that resilience and event centrality were significant mediators of positive (PTG) but not negative (PTS) outcomes. Although the frequency of ACEs and PTS symptoms in this study was low, people can respond to any event in different ways (Seery et al., 2010) and so it is important to be mindful of support options, if appropriate. Attending to emotional resilience and identity factors may be useful to inform support for individuals who have been exposed to ACEs, should it be needed. This provides further evidential support to recent recommendations (e.g. Brooks et al., 2017) that encourage individuals to acknowledge a central event where a positive, decisive change was made. At the same time, resilience and event centrality may be a worthy support focus in clinical samples where more frequent exposures to ACEs are reported alongside more severe PTS symptoms (e.g. Carroll et al., 2017), although future research is needed to examine this possibility. In this case, interventions may be better able to support survivors towards recognising potential gains from their experiences.

\section{Conclusion}


RESILIENCE, EVENT CENTRALITY AND GROWTH

This study examined the influence of emotional resilience and event centrality on PTG and PTS subsequent to ACEs. Findings indicated that following ACEs, the treatment and management of emotional resilience and event centrality could lead to positive effects on psychological well-being. Therefore, potential clinical applications of these findings may be to integrate the development of resilience and event centrality as intervention targets to encourage survivors to find meaning in their experiences. Future studies should consider the use of a clinical sample and longitudinal methods to establish causality among the relationships identified in this study. This would enable researchers to better explore how event centrality and resilience influence PTS and PTG in a population who experience a high proportion of ACEs to see if similar relationships are observed. 


\section{References}

Aydin, B., Akbas, S., Turla, A., \& Dundar, C. (2016). Depression and post-traumatic stress disorder in child victims of sexual abuse: perceived social support as a protection factor. Nordic Journal of Psychiatry, 70(6), 418-423. https://doi.org/10.3109/08039488.2016.1143028

Barlow, M. R., Turow, R. E. G., \& Gerhart, J. (2017). Trauma appraisals, emotion regulation difficulties, and self-compassion predict posttraumatic stress symptoms following childhood abuse. Child Abuse \& Neglect, 65, 37-47. https://doi.org/10.1016/i.chiabu.2017.01.006

Berntsen, D., \& Rubin, D. C. (2007). When a trauma becomes a key to identity: Enhanced integration of trauma memories predicts posttraumatic stress disorder symptoms. Applied Cognitive Psychology, 21(4), 417-431. https://doi.org/10.1002/acp.1290

Bernard, J. D., Whittles, R. L., Kertz, S. J., \& Burke, P. A. (2015). Trauma and event centrality: Valence and incorporation into identity influence well-being more than exposure. Psychological Trauma: Theory, Research, Practice, and Policy, 7(1), 11-17.

https://doi.org/10.1037/a0037331

Brooks, M., Graham-Kevan, N., Lowe, M., \& Robinson, S. (2017). Rumination, event centrality, and perceived control as predictors of post-traumatic growth and distress: The Cognitive Growth and Stress model. British Journal of Clinical Psychology, 56(3), 286-302. https://doi.org/10.1111/bjc.12138

Brooks, M., Graham-Kevan, N., Robinson, S., \& Lowe, M. (2019). Trauma characteristics and posttraumatic growth: The mediating role of avoidance coping, intrusive thoughts, and social support. Psychological Trauma: Theory, Research, Practice, and Policy, 11(2), 232-238. https://doi.org/10.1037/tra0000372 
RESILIENCE, EVENT CENTRALITY AND GROWTH

Bruskas, D., \& Tessin, D. H. (2013). Adverse childhood experiences and psychosocial well-being of women who were in foster care as children. The Permanente Journal, 17(3), e131-141. https://doi.org/10.7812/TPP/12-121

Bryan, R. H. (2019). Getting to why: Adverse childhood experiences' impact on adult health. The Journal for Nurse Practitioners, 15(2), 153-157. https://doi.org/10.1016/i.nurpra.2018.09.012

Bufford, R. K., Sisemore, T. A., \& Blackburn, A. M. (2017). Dimensions of grace: Factor analysis of three grace scales. Psychology of Religion and Spirituality, 9(1), 56-69. https://doi.org/10.1037/rel0000064

Carroll, T. D., Currier, J. M., McCormick, W. H., \& Drescher, K. D. (2017). Adverse childhood experiences and risk for suicidal behavior in male Iraq and Afghanistan veterans seeking PTSD treatment. Psychological Trauma: Theory, Research, Practice, and Policy, 9(5), 583-586. https://doi.org/10.1037/tra0000250

Chapman, D. P., Whitfield, C. L., Felitti, V. J., Dube, S. R., Edwards, V. J., \& Anda, R. F. (2004). Adverse childhood experiences and the risk of depressive disorders in adulthood. Journal of Affective Disorders, 82(2), 217-225. https://doi.org/10.1016/i.jad.2003.12.013

Clausen J. A. (1995). Gender, contexts, and turning points in adults' lives. In P. Moen, G. H. Elder, \& K. Luscher (eds.). Examining lives in context: Perspectives on the ecology of human development (pp. 365-389). American Psychological Association.

Duan, W., Guo, P., \& Gan, P. (2015). Relationships among trait resilience, virtues, post-traumatic stress disorder, and post-traumatic growth. PLoS One, 10(5). https://doi.org/10.1371/journal.pone.0125707 
RESILIENCE, EVENT CENTRALITY AND GROWTH

Draucker, C. B., Martsolf, D. S., Roller, C., Knapik, G., Ross, R., \& Stidham, A. W. (2011). Healing from childhood sexual abuse: A theoretical model. Journal of Child Sexual Abuse, 20(4), 435-466. https://doi.org/10.1080/10538712.2011.588188

Easton, S. D., Coohey, C., Rhodes, A. M., \& Moorthy, M. V. (2013). Posttraumatic growth among men with histories of child sexual abuse. Child Maltreatment, 18(4), 211-220. https://doi.org/10.1177/1077559513503037

Felitti, V. J., Anda, R. F., Nordenberg, D., Williamson, D. F., Spitz, A. M., Edwards, V., ... \& Marks, J. S. (1998). Relationship of childhood abuse and household dysfunction to many of the leading causes of death in adults: The Adverse Childhood Experiences (ACE) Study. American Journal of Preventive Medicine, 56(6), 245-258. https://doi.org/10.1016/S0749-3797(98)00017-

Foster, J. M. (2018). Resiliency, positive coping and posttraumatic growth in survivors of child abuse and neglect. In A. Kumar P., T. S. George, Sudesh N. T. (eds.), Character Strength Development: Perspectives from Positive Psychology (pp. 132-150). Sage Publishing.

Frampton, N. M., Poole, J. C., Dobson, K. S., \& Pusch, D. (2018). The effects of adult depression on the recollection of adverse childhood experiences. Child Abuse \& Neglect, 86, 45-54. https://doi.org/10.1016/i.chiabu.2018.09.006

Goodman, M. L., Gutarra, C., Billingsley, K. M., Keiser, P. H., \& Gitari, S. (2017). Childhood exposure to emotional abuse and later life stress among Kenyan women: a mediation analysis of cross-sectional data. Anxiety, Stress, \& Coping, 30, 469-483. https://doi.org/10.1080/10615806.2016.1271876

Groleau, J. M., Calhoun, L. G., Cann, A., \& Tedeschi, R. G. (2013). The role of centrality of events in posttraumatic distress and posttraumatic growth. Psychological Trauma: Theory, Research, Practice, and Policy, 5(5), 477-483. https://doi.org/10.1037/a0028809 
RESILIENCE, EVENT CENTRALITY AND GROWTH

Halligan, S. L., Michael, T., Clark, D. M., \& Ehlers, A. (2003). Posttraumatic stress disorder following assault: The role of cognitive processing, trauma memory, and appraisals. Journal of Consulting and Clinical Psychology, 71, 419-431. https://doi.org/10.1037/0022$\underline{006 X .71 .3 .419}$

Hayes, A. F. (2013). Introduction to mediation, moderation, and conditional process analysis: A regression-based approach. Guilford Press.

Infurna, F. J., \& Jayawickreme, E. (2019). Fixing the growth illusion: New directions for research in resilience and posttraumatic growth. Current Directions in Psychological Science, 28(2), 152158. https://doi.org/10.1177/0963721419827017

Jayawickreme, E., \& Blackie, L. E. (2014). Post-traumatic growth as positive personality change: Evidence, controversies and future directions. European Journal of Personality, 28(4), 312331. https://doi.org/10.1002/per.1963

Kalmakis, K. A., \& Chandler, G. E. (2015). Health consequences of adverse childhood experiences: A systematic review. Journal of the American Association of Nurse Practitioners, 27(8), 457465. https://doi.org/10.1002/2327-6924.12215

Kalmakis, K. A., Chiodo, L. M., Kent, N., \& Meyer, J. S. (2019). Adverse childhood experiences, posttraumatic stress disorder symptoms, and self-reported stress among traditional and nontraditional college students. Journal of American College Health, 1-8. https://doi.org/10.1080/07448481.2019.1577860

Kaloeti, D. V. S., Rahmandani, A., Sakti, H., Salma, S., Suparno, S., \& Hanafi, S. (2019). Effect of childhood adversity experiences, psychological distress, and resilience on depressive symptoms among Indonesian university students. International Journal of Adolescence and Youth, 24(2), 177-184. https://doi.org/10.1080/02673843.2018.1485584 
RESILIENCE, EVENT CENTRALITY AND GROWTH

Kerig, P. K., Bennett, D. C., Thompson, M., \& Becker, S. P. (2012). “Nothing really matters": Emotional numbing as a link between trauma exposure and callousness in delinquent youth. Journal of Traumatic Stress, 25(3), 272-279. https://doi.org/10.1002/jts.21700

Kilpatrick, D. G., Resnick, H. S., Milanak, M. E., Miller, M. W., Keyes, K. M., \& Friedman, M. J. (2013). National estimates of exposure to traumatic events and PTSD prevalence using DSM-IV and DSM-5 criteria. Journal of Traumatic Stress, 26(5), 537-547. https://doi.org/10.1002/jts.21848

Langford, D. J., Theodore, B. R., Balsiger, D., Tran, C., Doorenbos, A. Z., Tauben, D. J., \& Sullivan, M. D. (2018). Number and type of post-traumatic stress disorder symptom domains are associated with patient-reported outcomes in patients with chronic pain. The Journal of Pain, 19(5), 506-514. https://doi.org/10.1016/i.jpain.2017.12.262

Levine, S. Z., Laufer, A., Stein, E., Hamama-Raz, Y., \& Solomon, Z. (2009). Examining the relationship between resilience and posttraumatic growth. Journal of Traumatic Stress, 22(4), 282-286. https://doi.org/10.1002/jts.20409

Machisa, M. T., Christofides, N., \& Jewkes, R. (2016). Structural pathways between child abuse, poor mental health outcomes and male-perpetrated intimate partner violence (IPV). PloS One, 11(3). https://doi.org/10.1371/journal.pone.0150986

Meng, X., Fleury, M. J., Xiang, Y. T., Li, M., \& D’arcy, C. (2018). Resilience and protective factors among people with a history of child maltreatment: A systematic review. Social Psychiatry and Psychiatric Epidemiology, 53(5), 453-475. https://doi.org/10.1007/s00127-018-1485-2

Merrick, M. T., Ports, K. A., Ford, D. C., Afifi, T. O., Gershoff, E. T., \& Grogan-Kaylor, A. (2017). Unpacking the impact of adverse childhood experiences on adult mental health. Child Abuse \& Neglect, 69, 10-19. https://doi.org/10.1016/i.chiabu.2017.03.016 
RESILIENCE, EVENT CENTRALITY AND GROWTH

Monnat, S. M., \& Chandler, R. F. (2015). Long-term physical health consequences of adverse childhood experiences. The Sociological Quarterly, 56(4), 723-752. https://doi.org/10.1111/tsq.12107

Nillni, Y. I., Shayani, D. R., Finley, E., Copeland, L. A., Perkins, D. F., \& Vogt, D. S. (2020). The impact of posttraumatic stress disorder and moral injury on women veterans' perinatal outcomes following separation from military service. Journal of Traumatic Stress. Advance online publication. https://doi.org/10.1002/jts.22509

Olsson, L., Jerneck, A., Thoren, H., Persson, J., \& O’Byrne, D. (2015). Why resilience is unappealing to social science: Theoretical and empirical investigations of the scientific use of resilience. Science Advances, 1. https://doi.org/10.1126/sciadv.1400217

Preacher, K. J., \& Hayes, A. F. (2004). SPSS and SAS procedures for estimating indirect effects in simple mediation models. Behavior Research Methods, 36, 717-731. https://doi.org/10.3758/BF03206553

Preacher, K. J., \& Kelley, K. (2011). Effect size measures for mediation models: Quantitative strategies for communicating indirect effects. Psychological Methods, 16(2), 93-115. https://doi.org/10.1037/a0022658

Rawlins, B., Brooks, M., \& Khan, R. (in press). Posttraumatic stress symptoms mediate the relationship between adverse childhood experiences, avoidant personality traits and resilience. Anxiety, Stress, \& Coping.

Robinaugh, D. J., \& McNally, R. J. (2011). Trauma centrality and PTSD symptom severity in adult survivors of childhood sexual abuse. Journal of Traumatic Stress, 24(4), 483-486. https://doi.org/10.1002/jts.20656 
RESILIENCE, EVENT CENTRALITY AND GROWTH

Rutter, M. (2013). Annual research review: Resilience-clinical implications. Journal of Child Psychology and Psychiatry, 54(4), 474-487. https://doi.org/10.1111/j.1469$\underline{7610.2012 .02615 . x}$

Schaefer, L. M., Howell, K. H., Schwartz, L. E., Bottomley, J. S., \& Crossnine, C. B. (2018). A concurrent examination of protective factors associated with resilience and posttraumatic growth following childhood victimization. Child Abuse \& Neglect, 85, 17-27. https://doi.org/10.1016/j.chiabu.2018.08.019

Schalinski, I., Teicher, M. H., Nischk, D., Hinderer, E., Müller, O., \& Rockstroh, B. (2016). Type and timing of adverse childhood experiences differentially affect severity of PTSD, dissociative and depressive symptoms in adult inpatients. BMC Psychiatry, 16, 295. https://doi.org/10.1186/s12888-016-1004-5

Seery, M. D., Holman, E. A., \& Silver, R. C. (2010). Whatever does not kill us: cumulative lifetime adversity, vulnerability, and resilience. Journal of Personality and Social Psychology, 99(6), 1025-1041. https://doi.org/10.1037/a0021344

Shakespeare-Finch, J., \& Lurie-Beck, J. (2014). A meta-analytic clarification of the relationship between posttraumatic growth and symptoms of posttraumatic distress disorder. Journal of Anxiety Disorders, 28(2), 223-229. https://doi.org/10.1016/i.janxdis.2013.10.005

Sheffler, J. L., Stanley, I., \& Sachs-Ericsson, N. (2020). ACEs and mental health outcomes. In G. J. G. Admundson \& T. O. Afifi (eds.), Adverse Childhood Experiences (pp. 47-69). Academic Press. https://doi.org/10.1016/B978-0-12-816065-7.00004-5

Sheridan, G., \& Carr, A. (2020). Survivors' lived experiences of posttraumatic growth after institutional childhood abuse: An interpretative phenomenological analysis. Child Abuse \& Neglect, 103, 104430. https://doi.org/10.1016/i.chiabu.2020.104430 
RESILIENCE, EVENT CENTRALITY AND GROWTH

Stainton, A., Chisholm, K., Kaiser, N., Rosen, M., Upthegrove, R., Ruhrmann, S., \& Wood, S. J. (2019). Resilience as a multimodal dynamic process. Early Intervention in Psychiatry, 13(4), 725-732. https://doi.org/10.1111/eip.12726

Sutin, A. R., Costa Jr, P. T., Wethington, E., \& Eaton, W. (2010). Perceptions of stressful life events as turning points are associated with self-rated health and psychological distress. Anxiety, Stress, \& Coping, 23(5), 479-492. https://doi.org/10.1080/10615800903552015

Tabachnick, B. G., \& Fidell, L. S. (2013). Using multivariate statistics (Vol. 6). Pearson Education Limited.

Tavernier, R., \& Willoughby, T. (2012). Adolescent turning points: The association between meaningmaking and psychological well-being. Developmental Psychology, 48(4), 1058-1068. https://doi.org/10.1037/a0026326

Tedeschi, R. G., \& Calhoun, L. G. (2004). " Posttraumatic growth: Conceptual foundations and empirical evidence". Psychological Inquiry, 15(1), 1-18. https://doi.org/10.1207/s15327965pli1501 01

Thompson, N. J., Fiorillo, D., Rothbaum, B. O., Ressler, K. J., \& Michopoulos, V. (2018). Coping strategies as mediators in relation to resilience and posttraumatic stress disorder. Journal of Affective Disorders, 225, 153-159. https://doi.org/10.1016/i.jad.2017.08.049

Thomson, P., \& Jaque, S. V. (2017). Adverse childhood experiences (ACE) and adult attachment interview (AAI) in a non-clinical population. Child Abuse \& Neglect, 70, 255-263. https://doi.org/i.chiabu.2017.06.001

Vieselmeyer, J., Holguin, J., \& Mezulis, A. (2017). The role of resilience and gratitude in posttraumatic stress and growth following a campus shooting. Psychological Trauma: Theory, Research, Practice, and Policy, 9(1), 62-69. https://doi.org/10.1037/tra0000149 
RESILIENCE, EVENT CENTRALITY AND GROWTH

Weber, M. C., Pavlacic, J. M., Gawlik, E. A., Schulenberg, S. E., \& Buchanan, E. M. (2019). Modeling resilience, meaning in life, posttraumatic growth, and disaster preparedness with two samples of tornado survivors. Traumatology. Advance online publication. https://doi.org/10.1037/trm0000210

Westfall, N. C., \& Nemeroff, C. B. (2018). Child abuse and neglect as risk factors for post-traumatic stress disorder. In C. B. Nemeroff \& C. Marmar (eds.), Post-traumatic stress disorder (pp. 443-470). New York: Oxford University Press.

https://doi.org/10.1093/med/9780190259440.001.0001

Young, D. A., Neylan, T. C., O'Donovan, A., Metzler, T., Richards, A., Ross, J. A., \& Inslicht, S. S. (2018). The interaction of BDNF Val66Met, PTSD, and child abuse on psychophysiological reactivity and HPA axis function in a sample of Gulf War Veterans. Journal of Affective Disorders, 235, 52-60. https://doi.org/10.1016/j.jad.2018.04.004

Zeidner, M., \& Kampler, S. (2020). Memory traces of childhood exposure to terror attack and resilience and post-traumatic growth in adulthood. Personality and Individual Differences, 155, 109719. https://doi.org/10.1016/i.paid.2019.109719 
Tables

Table 1. Frequency scores of childhood maltreatment and household dysfunction experienced by participants.

\begin{tabular}{|c|c|c|}
\hline ACE & $N$ & $\%$ \\
\hline \multicolumn{3}{|l|}{ Did a parent or other adult in the household often or very often... } \\
\hline 1. Swear at you, insult you, put you down or humiliate you? & 33 & 19.8 \\
\hline 2. Act in a way that you might be physically hurt? & 32 & 19.2 \\
\hline 3. Push, grab, slap or throw something at you? & 32 & 19.2 \\
\hline 4. Ever hit you so hard that you had marks or were injured? & 29 & 17.4 \\
\hline \multicolumn{3}{|l|}{ Did an adult or person at least five years older than you... } \\
\hline 5. Touch or fondle you or have you touch their body in a sexual way? & 24 & 14.4 \\
\hline 6. Attempt to or actually have oral, anal, vaginal intercourse with you? & 16 & 9.6 \\
\hline \multicolumn{3}{|l|}{ Did you very often feel that... } \\
\hline 7. No-one in your family loved you or thought you were important/special? & 37 & 22.2 \\
\hline 8. Your family didn't look out for, feel close to, or support each other? & 39 & 23.4 \\
\hline $\begin{array}{l}\text { 9. You didn't have enough to eat, had to wear dirty clothes and had no-one to } \\
\text { protect you? }\end{array}$ & 0 & 0.0 \\
\hline $\begin{array}{l}\text { 10. Your parents were too drunk or high to take care of you or take you to the } \\
\text { doctor if you needed it? }\end{array}$ & 1 & 0.6 \\
\hline \multicolumn{3}{|l|}{ 11. Were your parents ever separated or divorced? } \\
\hline \multicolumn{3}{|l|}{ Was your mother, stepmother, father or stepfather... } \\
\hline $\begin{array}{l}\text { 12. Often or very often pushed, grabbed, slapped or had something thrown at } \\
\text { them? }\end{array}$ & 59 & 35.3 \\
\hline $\begin{array}{l}\text { 13. Ever repeatedly hit for at least a few minutes or threatened with a gun or } \\
\text { knife? }\end{array}$ & 1 & 0.6 \\
\hline $\begin{array}{l}\text { 14. Did you live with anyone who was a problem drinker or alcoholic or who used } \\
\text { street drugs? }\end{array}$ & 27 & 16.2 \\
\hline $\begin{array}{l}\text { 15. Was a household member depressed or mentally ill, or did a household } \\
\text { member attempt suicide? }\end{array}$ & 47 & 28.1 \\
\hline 16. Did a household member go to prison? & 3 & 1.8 \\
\hline
\end{tabular}


RESILIENCE, EVENT CENTRALITY AND GROWTH

Table 2. Mean and standard deviation of adverse childhood experiences, emotional resilience, event centrality, post-traumatic stress symptoms and post-traumatic growth.

\begin{tabular}{lcccccc}
\hline Variables & Mean & SD & Min. & Max. & Skew. & Kurt. \\
\hline Total ACE score & 2.37 & 2.87 & 0.00 & 12.00 & 1.22 & .59 \\
Emotional resilience & 27.54 & 8.05 & 0.00 & 40.00 & -.61 & .18 \\
Event centrality & 18.18 & 6.62 & 0.00 & 28.00 & -1.07 & -.21 \\
PTS symptoms & 3.74 & 1.66 & 0.00 & 5.00 & -.73 & .41 \\
PTG & 22.72 & 8.83 & 0.00 & 40.00 & -.26 & -.04 \\
\hline
\end{tabular}

Note. Skew. = skewness; Kurt. $=$ kurtosis 
RESILIENCE, EVENT CENTRALITY AND GROWTH

Table 3. Bivariate correlations between adverse childhood experiences, emotional resilience, event centrality, post-traumatic stress symptoms and post-traumatic growth.

\begin{tabular}{llllll}
\hline Variables & 1. & 2. & 3. & 4. & 5. \\
\hline 1. Total ACE score & - & $-.27^{* * *}$ & .14 & $-.73^{* * *}$ & .00 \\
2. Emotional resilience & & - & $.21^{* *}$ & $.25^{* *}$ & $.44^{* * *}$ \\
3. Event centrality & & - & $-.26^{* *}$ & $.52^{* * *}$ \\
4. PTS symptoms & & & - & -.11 \\
5. PTG & & & & - \\
\hline
\end{tabular}

${ }^{*} p<.05,{ }^{* *} p<.01,{ }^{* * *} p<.001$ 
Table 4.

Results of mediation analyses examining direct and indirect effects of ACE scores on PTS symptoms and PTG through resilience and event centrality.

\begin{tabular}{|c|c|c|c|c|c|c|c|c|}
\hline \multirow[b]{2}{*}{$\begin{array}{c}\text { Independent } \\
\text { variable }(X)\end{array}$} & \multirow[b]{2}{*}{ Mediator $(M)$} & \multicolumn{3}{|c|}{ Direct effect $\left(c^{\prime}\right)$} & \multicolumn{4}{|c|}{ Indirect effect $\left(a b_{c s}\right)$} \\
\hline & & $\beta$ & $S E$ & $p$ & $a b_{c s}$ & Boot SE & $\begin{array}{c}\text { Lower } 95 \% \\
\text { BCa Cl }\end{array}$ & $\begin{array}{c}\text { Upper } 95 \% \\
\mathrm{BCa} \mathrm{Cl}\end{array}$ \\
\hline \multicolumn{9}{|c|}{ PTS symptoms $(Y)$} \\
\hline \multirow{2}{*}{$\begin{array}{c}\text { Total ACE } \\
\text { score }\end{array}$} & Resilience & .10 & .01 & .068 & -.03 & .02 & -.07 & .00 \\
\hline & $\begin{array}{c}\text { Event } \\
\text { centrality }\end{array}$ & -.19 & .01 & .001 & -.03 & .01 & -.05 & .00 \\
\hline \multicolumn{9}{|c|}{ PTG $(Y)$} \\
\hline \multirow{2}{*}{$\begin{array}{c}\text { Total ACE } \\
\text { score }\end{array}$} & Resilience & .35 & .07 & $<.001$ & -.10 & .04 & -.17 & -.04 \\
\hline & $\begin{array}{c}\text { Event } \\
\text { centrality }\end{array}$ & .44 & .09 & $<.001$ & .06 & .03 & .01 & .12 \\
\hline
\end{tabular}

Notes. $S E$ = standard error; $95 \% \mathrm{BCa} \mathrm{Cl}=95 \%$ bias-corrected confidence interval; boldface values indicate significant effects $(p<.05)$; $c^{\prime}=$ standardized

direct effects of the independent variable on the dependent variable $(X \rightarrow Y)$ controlling for the mediator; $a b_{c s}=$ completely standardized indirect effects of the independent variable on the dependent variable via the mediator $(X \rightarrow M \rightarrow Y)$. 